\title{
Aprovechamiento de los Materiales de Cambio de Fase (PCM) en la Climatización
}

\author{
Manuel Domínguez y Carmen García \\ Instituto del Frío (CSIC), C/ Antonio Novais nº 10, 28040 Madrid-España \\ (e-mail: dominguez@if.csic.es; ifrg116@if.csic.es)
}

\begin{abstract}
Resumen
Se analizan las posibilidades que presentan los materiales de cambio de fase en la acumulación de grandes cantidades de calor en espacios reducidos en el campo de la climatización. Se describen diversas instalaciones de climatización en donde se han incorporado de forma satisfactoria los materiales de cambio de fase (PCM), y se estudian diversos tipos de instalaciones tanto con sistemas de producción de energía térmica, como las que aprovechan el frío gratis del aire de forma directa (free cooling) o indirecta mediante sistemas evaporativos, o los que aprovechan calores residuales o cogeneración. Considerando las múltiples posibilidades que presentan los PCM, se concluye que su empleo se generalizará en el futuro.
\end{abstract}

Palabras clave: materiales de cambio de fase, PCM, acumulación de calor, climatización, frío gratis

\section{Utilization of the Phase Change Materials (PCM) in the Air Conditioning}

\begin{abstract}
The possibilities that present phase change materials in the heat storage of big quantities of heat in reduced spaces in the field of the air conditioning are analyzed. Several installations of climatization are described where phase change materials (PCM) have been satisfactorily incorporated and diverse types of installations including systems of thermal energy production as well as those that take advantage of free cooling in direct form, or indirect form by means of evaporative systems, or those that take advantage of residual heats or cogeneration. Considering the multiple advantages of the PCM it is concluded that they will be widely used in the future.
\end{abstract}

Keywords: phase change materials, PCM, heat store, climatization, free cooling 


\section{INTRODUCCIÓN}

La subida tan importante de los precios del petróleo en los últimos tiempos, obliga a aumentar la eficiencia energética en las instalaciones, en particular las de mayor consumo. Unas de ellas es la climatización, entendiéndola en el sentido más amplio, es decir, incluyendo la calefacción. En muchos países las curvas de demanda eléctrica están pasando de los meses fríos a los más calurosos, debido precisamente a los consumos de las instalaciones de climatización. A la mayoría de los países, que no disponen de energía hidráulica suficiente para la producción de electricidad, con objeto de aplanar las curvas de demanda, les han obligado a cambiar a tarifas planas por otras diferenciales horarias, con valores muy variables de precio a lo largo del día y del año, siendo muy interesante la posibilidad de acumulación de calor obtenido en las horas de menor costo. En las centrales nucleares es muy complicada la regulación de capacidad y en las térmicas que emplean combustibles fósiles, es también un cierto problema, el ajuste de la producción de electricidad a las demandas.

Es importante tener también presente, que en las instalaciones de climatización, los máximos relativos de las curvas de demanda, corresponden a las horas de temperatura más altas del día, en las cuales los rendimientos de las instalaciones de climatización son desfavorables. Se debe tener en cuenta que, por cada grado Celsius que se baje la temperatura de condensación, se disminuye aproximadamente un $3 \%$ la producción de frío, es decir, trabajando en horas nocturnas se tiene dos importantes beneficios, uno de eficiencia energética y otro de costo de la electricidad. Esto se puede conseguir con la acumulación de frío.

Los sistemas de acumulación de frío empleados han sido: piscinas o tanques de agua y tanque de hielo. Los primeros conducen a grandes volúmenes, con sobrecostos, por problemas estructurales o pérdidas de superficie. La acumulación en bancos de hielo implica pérdidas de eficiencia, al tener que evaporar a temperaturas más bajas, en donde se pierde aproximadamente un $3 \%$ por grado de temperatura de evaporación. Aparte de la problemática de necesitar, el empleo de agua glicolada en lugar de agua, lo que aumenta mucho los consumos eléctricos en los sistemas de bombeo, por el aumento considerable de viscosidad. Se puede comprender que el empleo de materiales de cambio de fase a temperaturas más altas de las del hielo, tendrá muchas ventajas técnicas y conducirá a reducir los gastos de explotación muy considerablemente y aumentar la eficiencia en las instalaciones de climatización (Domínguez y Colubret, 2005)

Existen instalaciones de climatización singulares en las cuales se requieren fuentes de calor y de frío, hay otras que producen calores residuales importantes, y también hay otras en donde se podría aprovechar los calores de las propias máquinas, para cubrir las necesidades de agua caliente sanitaria (ACS) o para precalentar el agua en calderas de vapor. En general, se tiende a los aprovechamientos de las energías renovables disponibles, entre las que se encuentran: la solar, la del propio suelo o terreno y la del aire ambiente. Todas estas fuentes se podrán usar si se resuelve su gran problema, la estacionalidad o variabilidad en el tiempo. Es aquí donde los materiales de cambio de fase, conocidos internacionalmente por sus siglas en inglés como PCM, adquieren su importancia.

El margen de temperatura de cambio de fase de solidificación en la climatización, puede extenderse desde $0{ }^{\circ} \mathrm{C}$ hasta $100{ }^{\circ} \mathrm{C}$, siendo conveniente que presenten características, tales como: mucha energía en dicho cambio, que sean estables en el tiempo y con los ciclos, compatibles con los materiales que los contienen, sin problemas de toxicidad y de bajo costo. Teniendo en cuenta todos estos condicionantes y que la temperatura sea la adecuada, se reducen mucho los PCM disponibles. Zalba et al. (2003), describen los PCM que se emplean actualmente, mientras que Faustini (2007) lista abundante literatura sobre el tema.

En este trabajo se resumen y analizan algunas contribuciones relacionados con la mejora de la eficiencia energética en general y en particular con el empleo de estos materiales. Se presentan posibles aplicaciones, en particular el aprovechamiento del frío gratuito o como se le conoce internacionalmente "free cooling" que, junto con el enfriamiento evaporativo, tiene grandes posibilidades de uso en la climatización de casetas de telefonía, bodegas de crianza y naves 
industriales, polideportivos y edificios de oficinas. Se discuten también otros trabajos relacionados sobre el tema y la técnica de medida de las propiedades termofisicas de estos materiales, con cálculo de transmisión de calor empleando la analogía eléctrica resuelta con ordenador, optimización de grandes tanques de acumulación y utilización de microencapsaulados en circuitos de enfriamiento (Barragán et al., 2002; Domínguez et al., 2008). Finalmente se analizan trabajos recientes relacionados con sistemas de acumulación con estos materiales y métodos de cálculo de las instalaciones de climatización con estos materiales (Sharma et al., 2007; Gu et al., 2004), con optimización de su empleo en la construcción (Darkwa et al., 2006; Carbonari et al., 2006; Kuznik et al., 2008) y varias otras aplicaciones (Sarı y Karaipekli, 2008; Alkan et al., 2008).

\section{TIPOS DE INSTALACIONES DE CLIMATIZACIÓN}

Algunos tipos de instalaciones de climatización en donde la acumulación térmica es interesante y que se analizan en esta sección son: i) grandes centros comerciales o de oficinas; ii) bombas de calor domésticas; y iii) instalaciones con frío gratuito

\section{Grandes centros comerciales o de oficinas}

En estas aplicaciones se requiere una potencia térmica muy grande en los meses cálidos y en las horas de máxima temperatura del día. Conviene instalar pocas máquinas de producción de frío de gran potencia, pues son las que permiten COP elevados (del orden de 6). Estas máquinas presentan un especial problema: los tiempos de parada son grandes, del orden de una hora. En este caso, el acumulador de calor puede producir dos funciones: la de almacenar el frío producido por la noche y el de regular la capacidad de las máquinas. La incorporación del acumulador en el cierre del anillo primario presenta importantes ventajas; por ejemplo se carga cuando hay escasez de demanda y se descarga cuando hay gran demanda, pudiendo aumentar el caudal de los circuitos secundarios con respecto al primario. En la figura 1, se puede ver un esquema de dichas instalaciones en la que se han incluido las temperaturas usuales de diseño.

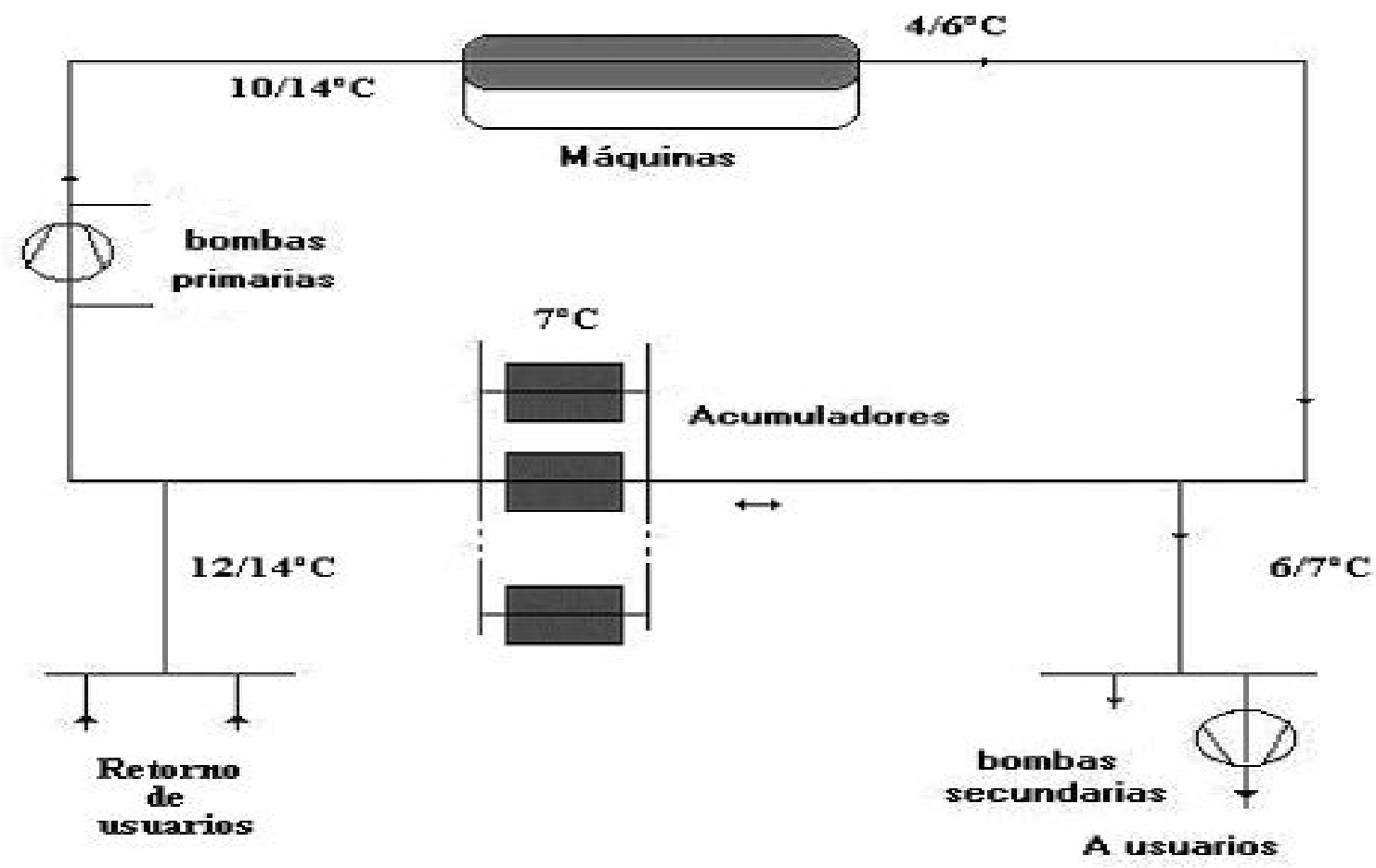

Fig. 1: Esquema de principio de una instalación de climatización con PCM en el lazo de cierre del circuito primario 
Se considera que en este tipo de instalación la temperatura más adecuada del cambio de fase del $\mathrm{PCM}$ es la de $7^{\circ} \mathrm{C}$, pudiendo trabajar en carga con saltos de 2 a $3^{\circ} \mathrm{C}$. Esto porque no conviene disminuir de $4^{\circ} \mathrm{C}$ la temperatura de salida de agua en las máquinas de enfriamiento, y en la descarga puede trabajarse entre 10 a $14^{\circ} \mathrm{C}$. Esto significa que los saltos entre el acumulador y el agua serian de $3^{\circ} \mathrm{C}$ en la carga y de $7^{\circ} \mathrm{C}$ en la descarga.

\section{Bombas de calor domésticas}

El empleo de las bombas de calor es ideal en climas que lo requieran en una estación y frío en otra y también en las que una de las estaciones sea muy larga. Las bombas de calor presentan problemas cuando la carga es pequeña con respecto a su capacidad. Los fabricantes están resolviendo estos inconvenientes con tanques de inercia de agua de capacidades entre 100 y 150 litros, que requieren espacios que los reducidos apartamentos actuales difícilmente disponen. La sustitución de dichos tanques por otros mucho más reducidos de volumen, entre 15 a 20 litros, pero incorporando PCM resolvería dichos problemas. Las temperaturas recomendadas también serian de $7^{\circ} \mathrm{C}$, si se coloca a la salida del enfriador o de $12^{\circ} \mathrm{C}$ sí es a la entrada del mismo. La acumulación tiene otra ventaja importante, ya que alarga los ciclos de funcionamiento-parada y con ello la vida de los compresores herméticos que tienen estas instalaciones. En la figura 2 se puede ver un símil hidráulico que facilita la comprensión de su funcionamiento. En la figura, Qo es el frío producido y Qc la carga térmica requerida.

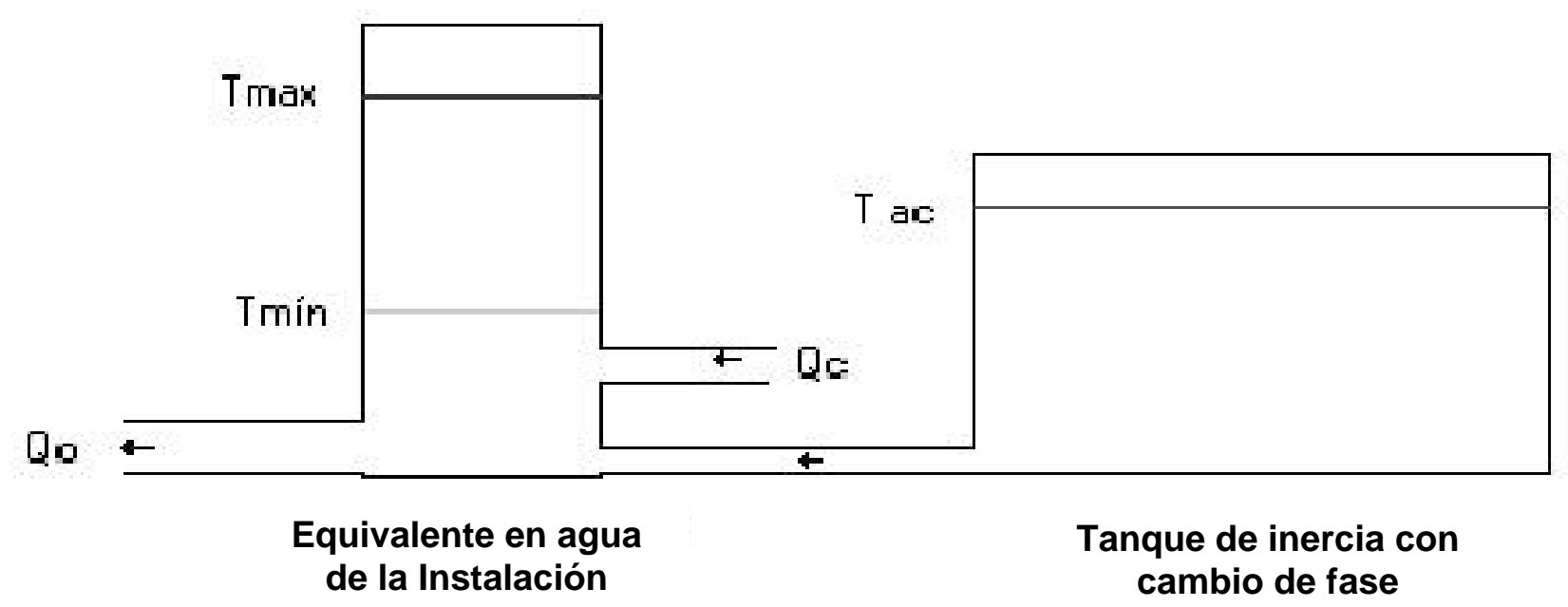

Fig. 2: Símil hidráulico de una instalación de bomba de calor con tanque de inercia térmica

\section{Instalaciones con frío gratuito}

El aprovechamiento del frío gratuito ("free cooling"), es un interesante concepto que se está empleando, con gran aumento la eficiencia energética, en muchas instalaciones singulares de climatización, como son las casetas de telefonía. Se considera que con su empleo se pueden eliminar, en ciertos casos, los sistemas clásicos de climatización (máquinas de compresión). Esto supone las siguientes ventajas: i) se disminuye los consumos energéticos; ii) se reduce y simplifica su mantenimiento; y iii) se reducen ruidos y vibraciones, de gran importancia en zonas urbanas. En la figura 3 se muestra un esquema de principio de una instalación con frío gratuito.

Durante la noche se permite la carga o solidificación del PCM y en el día, cuando la temperatura ambiente supere a la máxima deseada en la caseta, se evita que entre el aire del exterior. El aire recircula y se licua el PCM enfriando a la caseta. Con temperaturas de $26^{\circ} \mathrm{C}$ de cambio de fase, se puede mantener en un clima medio, con temperaturas en las casetas inferiores a $29^{\circ} \mathrm{C}$. En las Palmas (España) hay funcionando hace años una caseta de demostración. 


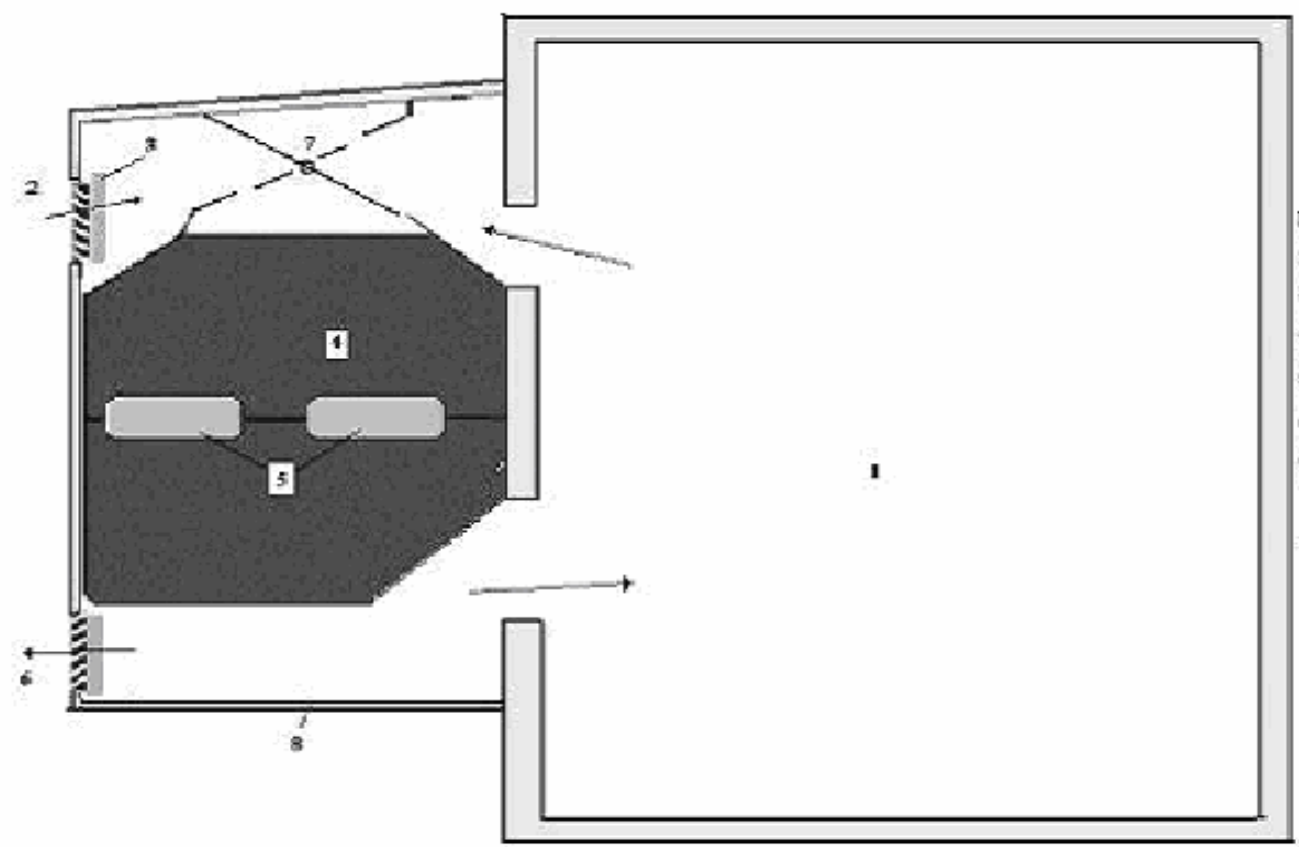

Fig. 3: Esquema de principio de una caseta de telefonía con PCM y frío gratis. 1 caseta; 2 toma de aire del exterior; 3 filtro de aire; 4 batería con el PCM; 5 ventiladores; 6 rejilla de salida del aire al exterior; 7 compuertas de regulación; 8 cofre aislado térmicamente.

Hay muchas otras aplicaciones que se ven frenadas por no bajar suficientemente la temperatura nocturna con respecto a la deseada de confort. En estos casos se puede acudir al enfriamiento evaporativo (Domínguez et al., 2002). En Galicia y Madrid están funcionando desde hace muchos años algunas casetas de telefonía con estos sistemas evaporativos y acumuladores PCM, con temperatura de $22^{\circ} \mathrm{C}$. En los enfriamientos evaporativos directos, hay casos que aumenta mucho la humedad. En estos casos se puede pensar en enfriamientos indirectos o intermitentes, como puede verse en los esquemas de la figura 4, empleando PCM.
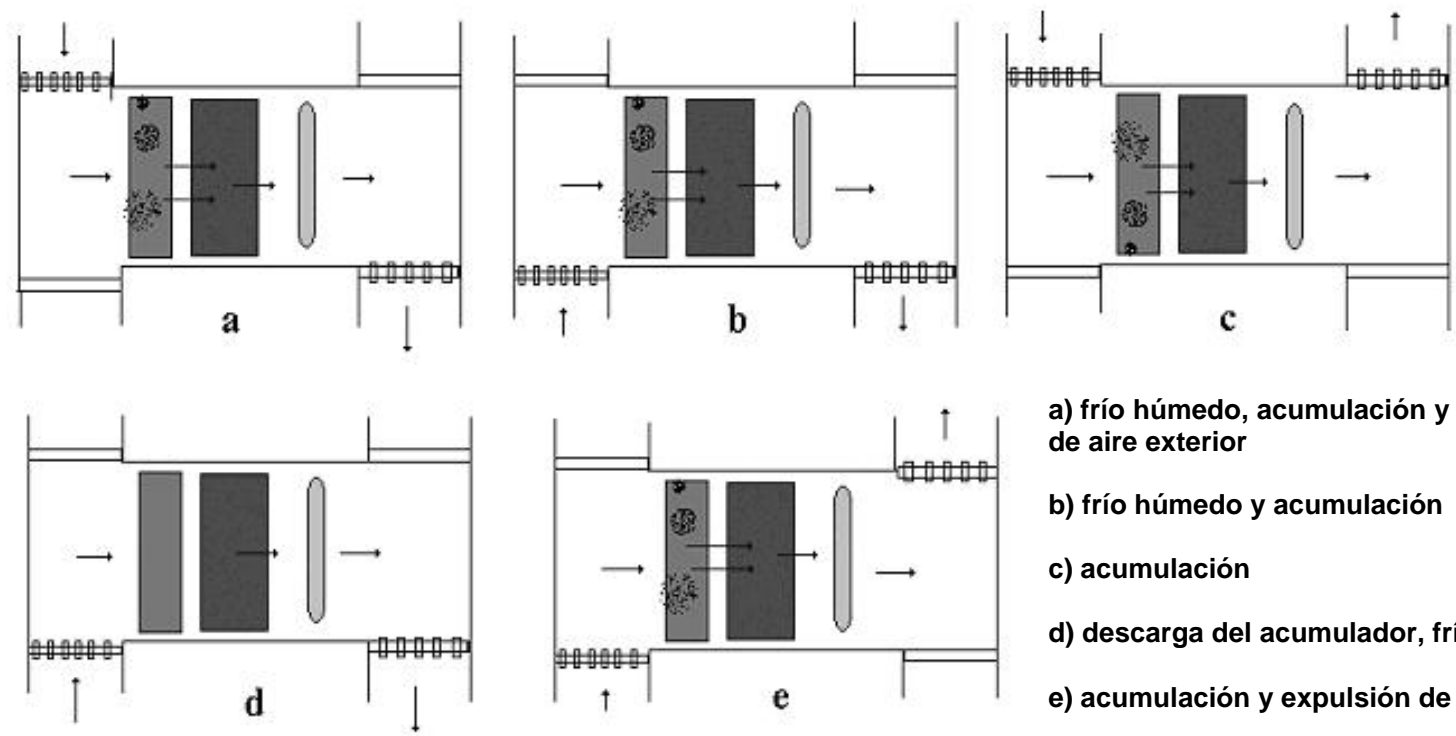

a) frío húmedo, acumulación y toma de aire exterior

b) frío húmedo y acumulación

c) acumulación

d) descarga del acumulador, frío seco

e) acumulación y expulsión de aire

Fig. 4: Esquemas de funcionamiento de un sistema evaporativo con PCM. Los elementos básicos son: cuatro rejillas regulables ( dos a dos antagónicas), un panel evaporativo, una bateria con el PCM y un ventilador 
En instalaciones prototipos ensayadas en clima húmedo como en Valencia, y en clima continental seco como Zaragoza, las temperaturas de cambio de fase de los materiales indicados en estos tipos de instalaciones, han sido de 26 y $23^{\circ} \mathrm{C}$, respectivamente. Las posibilidades de funcionamiento, pueden apreciarse en los cinco esquemas indicados en la figura 4. Como se puede observar, el sistema puede trabajar como recuperador de calor y evita el aumento de humedad interior, principal problema que los sistemas evaporativos directos. Se considera que en función de las temperaturas y de las humedades del aire exterior e interior, se podría, con un sistema evaporativo y un acumulador, climatizar naves industriales en climas continentales con ahorros energéticos muy importantes.

\section{DISCUSIÓN}

Los PCM se están desarrollando muy recientemente para ser aplicados en el transporte, en la construcción, en las aplicaciones solares y en la climatización, como se ha mostrado en este trabajo. Se considera que en un futuro se deben unir todas las aplicaciones y esfuerzos de las industrias químicas relacionadas con los PCM, para su mejor aprovechamiento. Se ha prestado más atención en este trabajo a la climatización por ser menos conocida.

Se considera por último, que sí se pudieran microencapsular estos materiales con precios no muy elevados, se podrían introducir en los circuitos de distribución de frío y de calor, es decir, hacer el transporte y la acumulación conjunta. En las instalaciones de frío hay tres temperaturas o niveles térmicos a los que se podría acumular energía térmica, el ya indicado de $7{ }^{\circ} \mathrm{C}$, el del calor de condensación, sobre los $40^{\circ} \mathrm{C}$, y el aprovechamiento del calor sensible a la salida de los compresores, del orden de $70^{\circ} \mathrm{C}$. En la figura 5 se ha representado el esquema de principio de una instalación con microencapsulados de PCM. Se estima que puede funcionar satisfactoriamente con PCM de hasta un $30 \%$ en volumen (Domínguez et al., 2008)

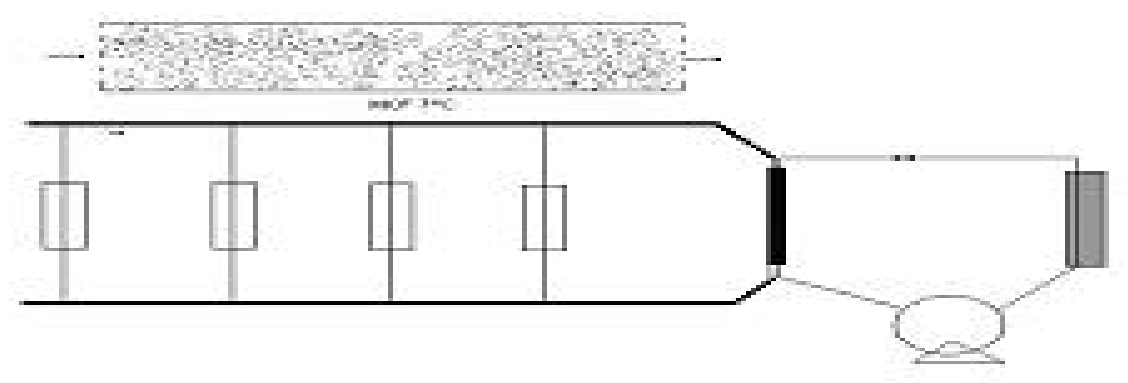

Fig. 5: Esquema ilustrativo de instalaciones de climatización con microencapsulación de PCM.

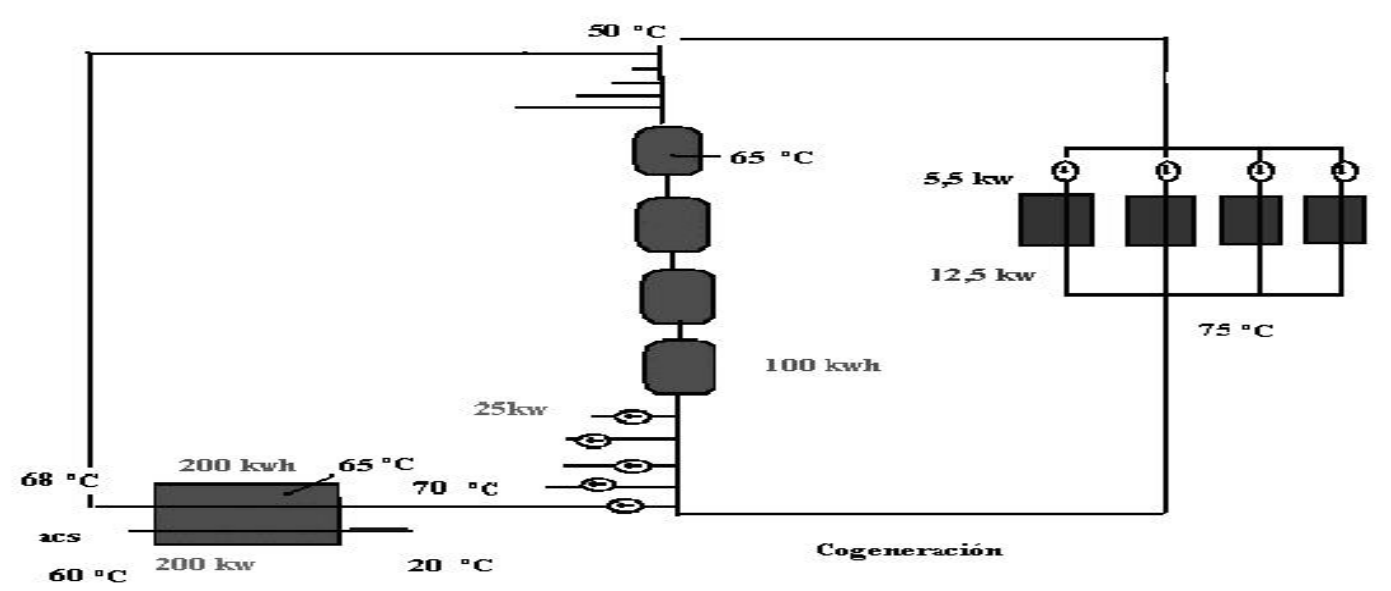

Fig. 6: Esquema de principio de un sistema de calefacción y ACS, con cuatro unidades de cogeneración. 
En estos momentos, en que la crisis energética aumenta, sé está tendiendo a desarrollar y aprovechar la cogeneración y el empleo de máquinas de absorción para aprovechar el calor residual. En estas instalaciones se requiere que su funcionamiento sea continuo, lo que obliga a la acumulación de calor a altas temperaturas, cerca de los $100^{\circ} \mathrm{C}$. En la figura 6 se indica, a modo ilustrativo, las potencias y temperaturas en que podría trabajar una de estas instalaciones para cubrir las necesidades de agua caliente sanitaria y de calefacción de un bloque de edificios para un clima como el de Madrid.

En la climatización se necesita mantener temperaturas en el amplio margen de 0 a 100 a $\mathrm{C}$, dependiendo de las aplicaciones. Afortunadamente, se disponen en el mercado de los PCM para este intervalo de temperatura a precios competitivos. Se está comenzando un desarrollo muy grande y de forma muy rápida, en la aplicación de estos materiales, como puede verse en los sistemas pasivos como los Muros tipo Trombe translucidos con el PCM, a temperaturas de 20,23 y $26{ }^{\circ} \mathrm{C}$ (figura 7).

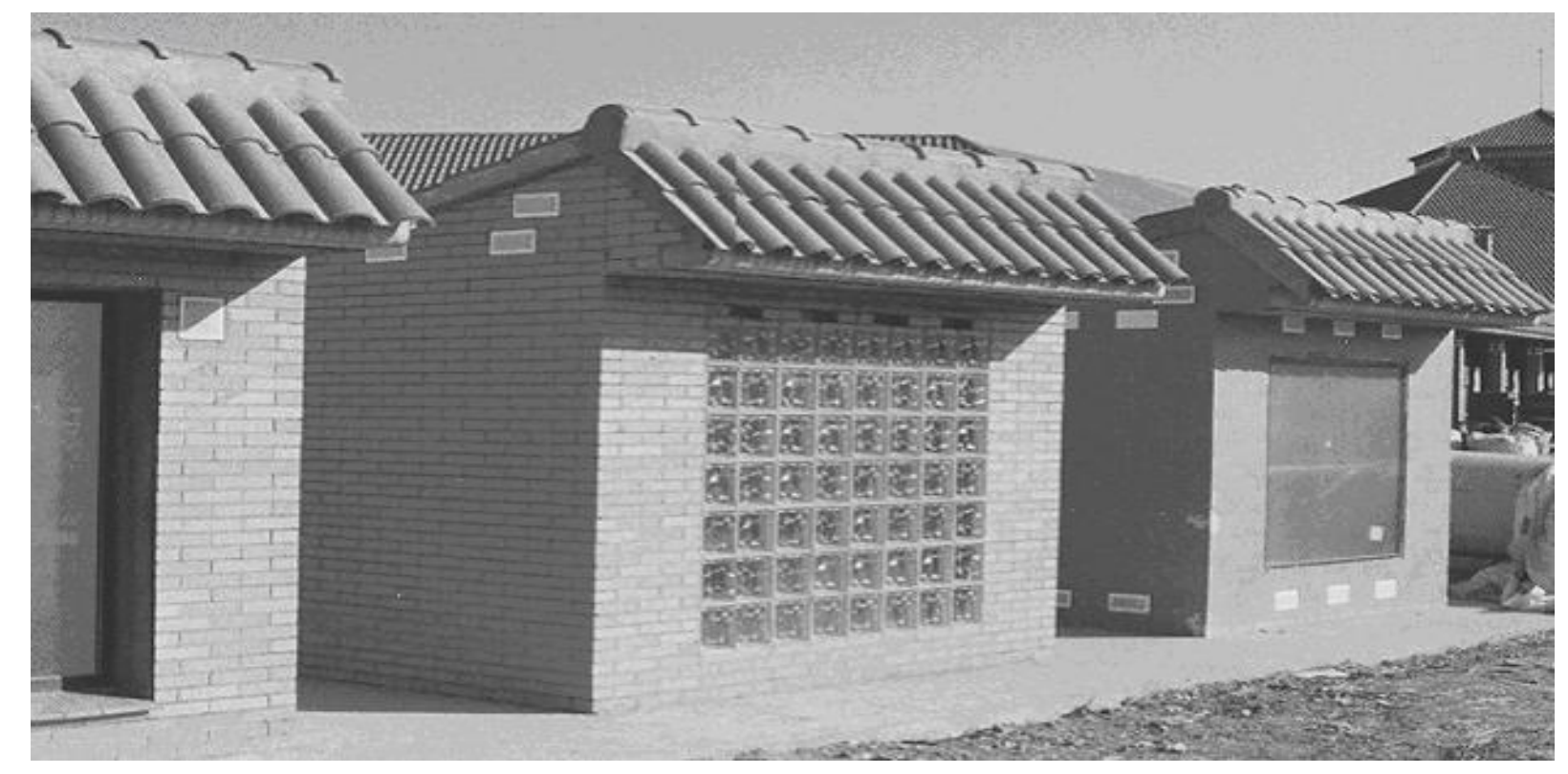

Fig. 7: Casetas donde se probaron los PCM de temperaturas 20, 23 y $26^{\circ} \mathrm{C}$. Toledo (España).

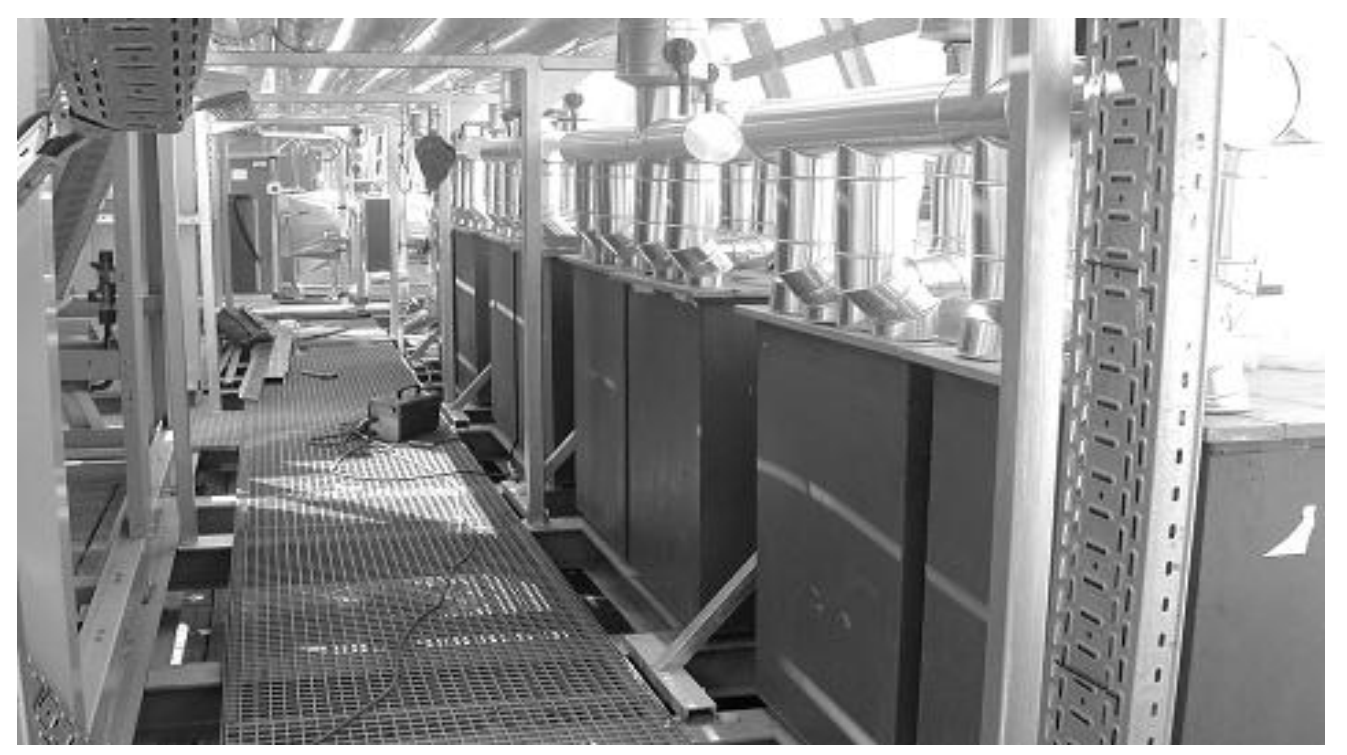

Fig. 8 Montaje de una instalación de climatización de $28 \mathrm{~m} 3$ de $\mathrm{PCM}$ de temperatura $7^{\circ} \mathrm{C}$. Madrid 
Existen en funcionamiento instalaciones de climatización con PCM de $6{ }^{\circ} \mathrm{C}$, con tanques de un metro cúbico que contienen dos baterías de cobre con aletas de aluminio, como se muestra en la figura 8 (Domínguez, 2006). Este sistema ha sido usado también en grandes instalaciones de climatización, como fue la realización en la ciudad del Banco de Santander de Madrid, con un volumen de PCM de $72 \mathrm{~m} 3$, con tanques iguales a los anteriores descritos. Actualmente sé está haciendo otra instalación en Sevilla del doble de capacidad, con tanques cilíndricos de plástico.

Se considera también de todo lo expuesto y analizado, que los PCM tienen un gran futuro en las instalaciones de climatización y que su uso masivo requerirá pasar por un periodo de desarrollo y optimización de sistemas y materiales para reducir costos. Hay pues, diversos campos abiertos muy bien diferenciados: el del transporte de productos perecederos, la construcción (muros, suelos y techos), las aplicaciones solares, la climatización y la calefacción. Se considera que la unión de todos los sectores involucrados facilitará el rápido desarrollo.

\section{CONCLUSIONES}

De los conceptos presentados y analizados en este trabajo se pueden obtener las siguientes conclusiones principales:

i) Se puede mejorar mucho su eficiencia en las instalaciones de climatización con el empleo de los PCM, que tengan la temperatura de cambio de fase adecuada al nivel de acumulación deseada;

ii) Se necesitará para generalizar su empleo, estudios técnicos de nuevas soluciones, experimentación y estudios teóricos de transmisión de calor, y sobre todo de estudios de viabilidad económica;

iii) Se considera que los riesgos son reducidos y los beneficios grandes, dada la evolución de los costos de la energía;

iv) Se ve muy grandes posibilidades a los PCM en la construcción, climatización y en el transporte, eliminando las máquinas de producción de frío en grandes partes del mundo; y

v) Los PCM tienen especial aplicación en casetas de telefonía, bodegas de crianza, naves industriales, polideportivos y edificios de oficinas.

\section{REFERENCIAS}

Alkan C., K. Kaya y A. Sarı; Preparation and thermal properties of ethylene glycol distearate as a novel phase change material for energy storage, Materials Letters: 62, 1122-1125 (2008).

Barragán V.M , J.M. Arias, M. Domínguez y C. Garcia; Testing the computer assisted solution of the electrical analogy in heat transfer processes with a phase change which has an analytical solution. International Journal of Refrigeration IIF: 25(5), 552-537 (2002).

Carbonari A., M. De Grassi, C. Di Perna y P. Principi ; Numerical and experimental analyses of PCM containing sandwich panels for prefabricated walls, Energy and Buildings: 38(5), 472-483 (2006).

Darkwa K., P.W. O'Callaghan y D. Tetlow; Phase-Change drywalls in a passive-solar building. Applied Energy: 83(5), 425-435 (2006).

Domínguez M., J. Colubret y A. Soto; Nuevo sistema de climatización evaporativo y acumuladores con cambio de fase para nodos de comunicación, Montajes e Instalaciones: (358), 64-68 (2002).

Domínguez M. y J. Colubret; /l Eficiencia energética en las instalaciones de frío y de climatización. El Instalador. 126(418), 160 (2005).

Domínguez M.; Los PCM en la climatización y en la construcción. El instalador: 24(430), 31 (2006). 
Domínguez M., C. García y A. Viti; Los microencapsulados en las instalaciones de climatización. El Instalador: 12(452), 30 (2008).

Faustini C.; Análisis del aprovechamiento energético de los acumuladores de cambio de fase en algunas propuestas constructivas. Tesis Doctoral. Universidad Politécnica de Madrid. Escuela de Arquitectura (2007).

Gu Z., H. Liu e Y. Li; Thermal energy recovery of air conditioning system-heat recovery system calculation and phase change materials development. Applied Thermal Engineering: 24, 2511-2526 (2004).

Kuznik F., J. Virgone y J. Noel; Optimization of a phase change material wallboard for building use, Applied Thermal Engineering: 28(11-12), 1291-1298 (2008).

Sarı A. y Ali Karaipekli; Preparation and thermal properties of capric acid/palmitic acid eutectic mixture as a phase change energy storage material, Materials Letters: 62(6-7), 903-906 (2008).

Sharma A., V.V. Tyagi, C.R. Chen y D. Buddhi; Review on thermal energy storage with phase change materials and applications. Renewable and Sustainable Energy Reviews: In Press, Corrected Proof, Available online 17 Dec (2007).

Zalba B., J.M. Marín, L.F. Cabeza y H. Mehling; Review on thermal energy storage with phase change: materials, heat transfer analysis and applications, Applied Thermal Engineering: 23(3), 251283 (2003). 
